\title{
Über die Nucleinsäure aus den Spermatozoen des Hamo \\ (Muraenoesox cinereus Forsk). \\ Von \\ Katsuji Inouye.
}

(Aus dem medizinisch-chemischen Institut der Universität zu Kyoto.)

(Der Redaktion zugegangen am 16. Mai 1906.)

Im Anschluß an die vor kurzem von mir publizierte Arbeit, 1 ) die Zusammensetzung und die Spaltungsprodukte der Darmnucleinsäure betreffend, teile ich die Untersuchungen mit, welche an der Hamonucleinsäure ${ }^{2}$ ) angestellit wurden.

Zur Darstellung der Nucleinsäure bediente ich mich der reifen Spermatozoen des Hamo, Muraenoesox cinereus Forsk. Das Verfahren war das gleiche wie bei dèr Darmnucleinsäure. ${ }^{3}$ ) Die gereinigte Nucleinsäure stellt eine weißé amorphe, in Wasser fast unlösliche: Masse dar. Sie löst sich in Wasser bei Gegenwart von Natriumacetat, besonders leicht in Alkalien. Eine $5 \%$ ige Lösung, die mit Hilfe von Natriumacetat hergestellt ist, erstarrt beim Erkalten zu einer festen Gallerte.

Die Hamonucleinsäure läß̈t sich aus der alkalischen Lösung nicht durch Essigsäure, wohl aber durch Salzsäure ausfällen. Mit Metallen bildet sie Salze; die neutralen Alkalisalze sind in Wasser leicht löslich, während die Schwermetalle meist unlösliche Verbindungen geben. Die Lösung der Alkalisalze und die durch Zusatz von Natriumacetat hergestellte Lösung der freien Säure drehen die Ebene des polarisierten Lichtes nach rechits.

Die Hamonucleinsäure gibt nicht die Farbenreaktionen der Eiweißkörper. Sie erzeugt einen weißen Niederschlag in sauren Lösungen von Albumosen, welcher in verdümter Salzsäure fast unlöslieh ist:

1) Diese Zeitschrift, Bd. XiVI, S. 201.

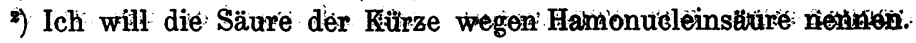

3) a. a. 0 .

Hoppe-Seyler's Zeitschrift f. physiol. Chemie. XLVIII. 


\section{Analyse der Hamonucleinsäure.}

Die Ausführung der Analyse erfolgte in derselben Weise wie bei Darmnucleinsäure.

$$
\begin{array}{r}
0,1581 \mathrm{~g} \text { Substanz gaben } 0,0620 \mathrm{~g} \mathrm{H}_{\mathrm{g}} \mathrm{O}=4,40 \% \mathrm{H} \\
\text { und } 0,2169 \mathrm{~g} \mathrm{CO}_{2}=37,42 \% \mathrm{C} \\
0,1757 \mathrm{~g} \text { Substanz gaben } 0,0658 \mathrm{~g} \mathrm{H}_{2} \mathrm{O}=4,19 \% \mathrm{H} \\
\text { und } 0,2418 \mathrm{~g} \mathrm{CO}_{2}=37,53 \% \mathrm{C} \\
0,2162 \mathrm{~g} \text { Substanz gaben } 0,0863 \mathrm{~g} \mathrm{H}_{2} \mathrm{O}=4,47 \% \mathrm{H} \\
\text { und } 0,2967 \mathrm{~g} \mathrm{CO}_{2}=37,43 \% \mathrm{C} \\
0,3532 \mathrm{~g} \text { Substanz gaben } 0,1358 \mathrm{~g} \mathrm{H}_{2} \mathrm{O}=4,31 \% \mathrm{H} \\
\text { und } 0,4873 \mathrm{~g} \mathrm{CO}_{2}=37,62 \% \mathrm{C} \\
0,2336 \mathrm{~g} \text { Substanz gaben } 0,0921 \mathrm{~g} \mathrm{H}_{2} \mathrm{O}=4,42 \% \mathrm{H} \\
\text { und } 0,3211 \mathrm{~g} \mathrm{CO}_{2}=37,49 \% \mathrm{C}
\end{array}
$$

$0,1777 \mathrm{~g}$ Substanz gaben $23,5 \mathrm{ccm}$ feuchten Stickstoffs bei $5,5^{\circ} \mathrm{C}$. und $759 \mathrm{~mm}$ B., entsprechend $16,13 \% \mathrm{~N}$.

$0,2085 \mathrm{~g}$ Substanz gaben $28 \mathrm{ccm}$ feuchten Stickstoffs bei $7^{\circ} \mathrm{C}$. und 748,7 mm B., entsprechend 16,06\% N.

$0,2053 \mathrm{~g}$ Substanz gaben $27 \mathrm{ccm}$ feuchten Stickstoffs bei $5,5^{\circ} \mathrm{C}$. und $765 \mathrm{~mm}$ B., entsprechend $16,16 \% \mathrm{~N}$.

0,2422 g Substanz gaben $31,5 \mathrm{ccm}$ feuchten Stickstoffs bei $5^{\circ} \mathrm{C}$. und $760 \mathrm{~mm}$ B., entsprechend 15,91\% N.

$0,1521 \mathrm{~g}$ Substanz gaben $20 \mathrm{ccm}$ feuchten Stickstoffs bei $5^{\circ} \mathrm{C}$. und $759 \mathrm{~mm} \mathrm{~B}$., entsprechend 16,06\% N.

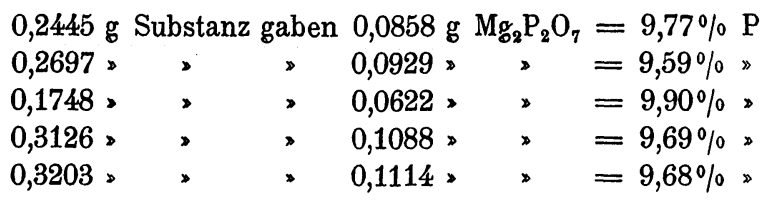

Es wurden gefunden:

\begin{tabular}{rrrrrrr} 
& 1 & 2 & 3 & 4 & 5 & Mittel \\
& \multicolumn{1}{c}{$\%$} & \multicolumn{1}{c}{$\%$} & \multicolumn{1}{c}{$\%$} & \multicolumn{1}{c}{$\%$} & \multicolumn{1}{c}{$\%$} & \multicolumn{1}{c}{$\%$} \\
$\mathrm{C}$ & 37,42 & 37,53 & 37,43 & 37,62 & 37,49 & 37,50 \\
$\mathrm{H}$ & 4,40 & 4,19 & 4,47 & 4,31 & 4,42 & 4,36 \\
$\mathrm{~N}$ & 16,13 & 16,06 & 16,16 & 15,91 & 16,06 & 16,04 \\
$\mathrm{P}$ & 9,77 & 9,59 & 9,90 & 9,69 & 9,68 & 9,73
\end{tabular}

Vergleicht man die gefundenen Analysenwerte mit den Zahlen: C 37,54\%, H 4,82\%, M 15,53\%, P 9,37\%, welche ich bei den Analysen der Darmnucleinsäure erhalten habe, so läßt sich nicht verkennen, daß die Zusammensetzung der Hamonucleinsäure die weitgehende Ähnlichkeit mit derjenigen der Darmnucleinsäure zeigt. 


\section{Spaltung der Hamonucleinsäure.}

$70 \mathrm{~g}$ hamonucleinsaures Natron wurden, wie bei Darmnucleinsäure vorgegangen war, mit $700 \mathrm{ccm}$ 20-volumprozentiger Schwefelsäure 12 Stunden auf dem Sandbade erhitzt, sodann von der abgeschiedenen Huminsubstanz abfiltriert und zu wiederholten Malen mit Äther ausgeschüttelt.

Der ätherlösliche Teil wurde nicht weiter untersucht, da ich bereits erwiesen hatte, $\left.{ }^{1}\right)$ daß die Lävulinsäure den wesentlichen Bestandteil desselben bildet.

Der ätherunlösliche Teil wurde mit überschüssigem Baryumcarbonat gekocht, filtriert, das Filtrat auf den Gehalt von 5-volumprozentiger Schwefelsäure gebracht und mit Phosphorwolframsäure ausgefällt.

\section{A. Phosphorwolframsäureniederschlag.}

Der Niederschlag wurde nach der Zerlegung mit Barythydrat und der Entfernung des überschüssigen Baryts durch Kohlensäure genau nach dem bei der Darmnucleinsäure beschriebenen Verfahren $^{2}$ ) auf Purinbasen und Cytosin verarbeitet. Es wurden gefunden: 0,8231 $\mathrm{g}$ Guanin, $1,6238 \mathrm{~g}$ Adenin, 0,1211 $\mathrm{g}$ Xanthin, $0,2410 \mathrm{~g}$ Hypoxanthin und 2,3911 $\mathrm{g}$ Cytosin.

1. Guanin. Das Guanin wurde als Sulfat analysiert. $0,1288 \mathrm{~g}$ Substanz gaben $36,6 \mathrm{ccm}$ feuchten Stickstoffs bei $5,5^{\circ} \mathrm{C}$. und $768,8 \mathrm{~mm} \mathrm{~B}$., entsprechen $35,10 \% \mathrm{~N}$.

Berechnet für $\left(\mathrm{C}_{6} \mathrm{H}_{6} \mathrm{~N}_{6} \mathrm{O}_{8} \mathrm{H}_{8} \mathrm{SO}_{4}\right.$ : $35,05 \% \mathrm{~N}$.

Gefunden: $35,10 \% \mathrm{~N}$.

2. Adenin. Das durch Umkrystallisation gereinigte Adeninpikrat, welches die charakteristische Krystallform zeigte, lieferte bei den Analysen die folgenden Zahlen:

0,1862 g Substanz gaben $47,3 \mathrm{ccm}$ feuchten Stickstoffs bei $5,5^{\circ} \mathrm{C}$. und $756 \mathrm{~mm}$ B., entsprechend $30,85 \% \mathrm{~N}$.

$0,1800 \mathrm{~g}$ Substanz gaben $0,0370 \mathrm{~g} \mathrm{H}_{2} \mathrm{O}=2,30 \% \mathrm{H}$ und $0,2400 \mathrm{~g} \mathrm{CO}_{2}=36,36 \% \mathrm{C}$.

Berechnet für $\mathrm{C}_{6} \mathrm{H}_{6} \mathrm{~N}_{6} \cdot \mathrm{C}_{6} \mathrm{H}_{8} \mathrm{~N}_{8} \mathrm{O}_{7}$ : Gefunden: $36,26 \%$ C, $2,30 \%$ H, $30,82 \%$ N. $\quad 36,36 \%$ C, $2,30 \%$ H, $30,85 \%$ N.

3. Xanthin. Die Substanz, die die charakteristischen Reaktionen für das Xanthin gab, wurde in der bekannten Weise ins Nitrat übergeführt und dann zur Analyse verwendet.

1) Diese Zeitschrift, Bd. XLII, S. 117. - 2) a. a. 0. 
$0,1744 \mathrm{~g}$ Substanz gaben $46 \mathrm{cem}$ feuchten Stickstoffs bei $5^{\circ} \mathrm{C}$. und $7.69 \mathrm{~mm}$ B., entsprechend $32,65 \% \mathrm{~N}$.

Berechnet. für $\mathrm{C}_{8} \mathrm{H}_{4} \mathrm{~N}_{4} \mathrm{O}_{2} \cdot \mathrm{HNO}_{8}$ :

Gefunden: $32,56 \% \mathrm{~N}$. $32,65 \% \mathrm{~N}$.

4. Hypoxanthin. Das Hypoxanthinnitrat krystallisierte in Wetzsteinformen und erwies sich bei der Analyse als rein. $0,1722 \mathrm{~g}$ Substanz gaben $49 \mathrm{ccm}$ feuchten Stickstoffs bei $5,4^{\circ} \mathrm{C}$. und 767,3 mm B., entsprechend $35,08 \% \mathrm{~N}$ :

Berechnet für. $\mathrm{C}_{5} \mathrm{H}_{4} \mathrm{~N}_{4} \mathrm{O} \cdot \mathrm{HNO}_{8}$ :

Gefunden: $35,23 \% \mathrm{~N}$. $35 ; 08 \% \% \mathrm{~N}$ :

5. C.ytosin. Die Substanz war rein weiß und krystallisierte in Blättchen. Die Analysen lieferten die folgenden Werte:

$0,2512 \mathrm{~g}$ Substanz gaben $0,1035 \mathrm{~g} \mathrm{H}_{2} \mathrm{O}=4,62 \% \mathrm{H}$ und $0,3968 \mathrm{~g} \mathrm{CO}_{2}=43,11 \% \mathrm{C}$

$0,1753 \mathrm{~g}$ Substanz gaben $54 \mathrm{ccm}$ feuchten Stickstoffs bei $6^{\circ} \mathrm{C}$. und $764 \mathrm{~mm} \mathrm{~B}$., entsprechend $37,74 \% \mathrm{~N}$.

Berechnet für $\mathrm{C}_{4} \mathrm{H}_{5} \mathrm{~N}_{8} \mathrm{O}$ : Gefunden: $43,18 \% \mathrm{C}, 4,54 \% \mathrm{H}, 37,89 \% \mathrm{~N} \quad 43,11 \% \mathrm{C}, 4,62 \% \mathrm{H}, 37,74 \% \mathrm{~N}$.

B. Das Filtrat der Phosphorwolframsäurefällung.

Nach der Entfernung der Phosphorwolframsäure und Schwefelsäure durch Barythydrat und des überschüssigen Baryts durch Durchleiten von $\mathrm{CO}_{2}$ wurde die Flüssigkeit auf dem Wasserbade stark eingeengt. und stehen gelassen. Aus dieser Flüssigkeit ließ. sich ein in Blättchen krystallisierter Körper gewinnen, der alle Eigenschaften des Thymins besaß. Die Ausbeute an diesem Körper betrug 2,0188 g. Die Analysen gaben die folgenden Werte:

$$
\begin{array}{r}
0,2012 \mathrm{~g} \text { Substanz gaben } 0,0886 \mathrm{~g} \mathrm{H}_{2} \mathrm{O}=4,93 \% \mathrm{H} \\
\text { und } 0,3497 \mathrm{~g} \mathrm{CO}_{2}=47,40 \% \mathrm{C}
\end{array}
$$

$0,2277 \mathrm{~g}$ Substanz gaben $41,5 \mathrm{ccm}$ feuchten Stickstoffs bei $5^{\circ} \mathrm{C}$. und $758,5 . \mathrm{mm}$ B., entsprechend $22,25 \%$. N.

Berechnet für $\mathrm{C}_{5} \mathrm{H}_{8} \mathrm{~N}_{2} \mathrm{O}_{2}$ :

Gefunden:

$47,57 \%$ Gi $4,80 \% \mathrm{H}_{2}, 22,26 \%$. N. $\quad 47,40 \%$ C, $4,93 \% \mathrm{H}, 22,25 \% \mathrm{~N}$.

Der von mir erhaltene Körper war demnach das Thy min.

Bei der Hydrolyse mit Schwefelsäure ${ }^{1}$ ) lieferte die Famonucleinsäure: Lävulinsäure, Guanin, Adenin, Xanthin, Hypoxanthin, Cytosin und Thymin.

1) Daß unter den Spaltungsprodukten die Phosphorsäure vorhanden war, habe ich durch einen besonderen Versuch festgestellt. 